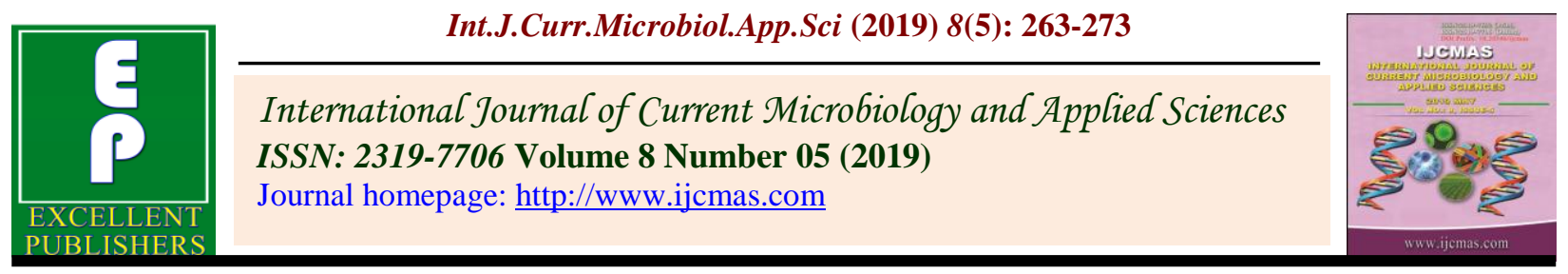

Original Research Article

https://doi.org/10.20546/ijcmas.2019.805.030

\title{
Consequence of Fym and Fly Ash Application on Yield, Nutrient Uptake, and Quality of Safed Musli Grown on Inceptisols
}

\author{
Payal B. Hadke", S.G. Wankhade and Sagar N. Ingle \\ Department of Soil Science and Agril. Chemistry, Dr. Panjabrao Deshmukh Krishi \\ Vidyapeeth, Akola- 444104, India \\ *Corresponding author
}

\begin{abstract}
A B S T R A C T
Effect of FYM and fly ash application on yield, content, uptake and quality of safedmusali in Inceptisols was investigated in field experiment carried out on clayey soil at

\begin{tabular}{|c|}
\hline Keywords \\
\hline $\begin{array}{l}\text { FYM, Fly-ash, } \\
\text { Safed Musali, } \\
\text { Saponin }\end{array}$ \\
\hline Article Info \\
\hline $\begin{array}{l}\text { Accepted: } \\
\text { 04 April } 2019 \\
\text { Available Online: } \\
\text { 10 May } 2019\end{array}$ \\
\hline
\end{tabular}
Nagarjun Medicinal Plants Garden, Dr. Panjabrao Deshmukh Krishi Vidyapeeth, Akola (M.S.). There were three levels of FYM and four levels of fly ash with three replications tried in Factorial randomised block design. The results obtained in the present investigation showed that, the number of leaves per plant and leaf area of Safed musli were non significantly influenced due to application of Fly ash. No significant effect of FYM application was also noticed on number of leaves. However, leaf area was significantly influenced with the application of FYM upto $10 \mathrm{t} \mathrm{FYM} \mathrm{ha}^{-1}$. The fresh as well as dry root yield was significantly recorded highest with the application of $20 \mathrm{t} \mathrm{FYM} \mathrm{ha}^{-1}$. The highest safed musli root yield was also recorded with the application of fly ash $15 \mathrm{t} \mathrm{ha}^{-1}$. However, it was at par with $10 \mathrm{t}$ and $5 \mathrm{t} \mathrm{ha}^{-1} \mathrm{Fly}$ ash applications. The content and uptake of nutrients, were also significantly influenced with the application of FYM and fly ash. The saponin content and yield and protein content of Safed musli roots were significantly increased with $20 \mathrm{t} \mathrm{FYM} \mathrm{ha}^{-1}$. The significantly highest saponin content and yield and protein content also recorded with $15 \mathrm{t}$ Fly ash $\mathrm{ha}^{-1}$. However, it was at par with 10 and $5 \mathrm{t}$ fly ash levels.
\end{abstract}

\section{Introduction}

Safed musli (Chlorophytum borivililanum) is an important medicinal perennial herb, belongs to family Liliaceae, widely distributed in India and found in hilly regions of Himalay, Satpuda, Vindhay, Aravuli and in hilly area of Bihar and Assam. It is also found in the part of Rajasthan and Gujarat. In Maharashtra, particularly, in Vidarbha, safed musli found widely in the forest of Melghat, Chikhaldara and Satpuda hills nearby Akot
(Akola) and Jalgaon Jamod (Buldhana). This species has low rate of regeneration due to shy flowering and poor setting of viable seed. It may thus become rare and may be lost from the habitat if the large scale collection in forest continues at the present rate (Bordia, 1992).

The fasciculated root of this herb has great medicinal values mainly due to its saponin content and is used extensively in Ayurvedic medicines. The roots also having aphrodisiac 
properties and therefore it is an important ingredient of herbal tonic to cure general debility and male sterility (Kirtikar and Basu, 1975). Bordia et al., (1995) reported that the major constituents of safed musli are carbohydrates $(42 \%)$, protein $(8-9 \%)$, root fibres (3-4\%) and saponin (2-17\%). Presently, the root fetches an attractive price in the market, which is mainly determined by the size and colour of the roots and its physical appearance. For obtaining good quality roots, the plant growth medium should be porous with optimum fertility status. In that context; the application of FYM and fly ash might be best source for improving physical properties of soil as well as provide nutrients to the crop.

Productivity is greatly influenced by the pattern of rainfall distribution, soil type, soil nutrient status, temperature and climatic factors. The high cost of fertilizer and low purchasing capacity of the small and marginal peasants of the country which restrict the use of costly fertilizer inputs.

About 130 coal-based thermal power stations in India are producing over165 million tons fly ash per year (CEA report 2014). With the increasing demand of power, more thermal power stations are expected to be commissioned or increase expected in their existing capacity (Mukherjee and Vesmawala, 2013).

Fly ash is a by-product of the Thermal Power Station, where coal energy is converted into electrical energy. The demand for electrical energy is increasing day by day and in the absence of its utilization of the byproduct of thermal plant, it is been getting accumulated and for storing large area of arable land will be required which is therefore finding immediate use of fly ash for different purposes is very important. Fly ash being an inert heterogeneous $\mathrm{SiO}_{2}, \mathrm{Al}_{2} \mathrm{O}_{3}$ and $\mathrm{Fe}_{2} \mathrm{O}_{3}$ as a main components and material containing mineral nutrients as minor components which attracts the agriculture scientists for its utilization in improving crop and soil productivity.

A part from nutrition fly ash generation is expected to increase to 300 million tons per annum by 2017 and 900 million tons per annum by 2031-32 (Planning Commission, 2014) it is a fine, grey, amorphous powder and rich in $\mathrm{Si}, \mathrm{Al}$, spherical in shape having excellent physical properties.

The physic-chemical properties of fly ash may vary widely, depending upon the nature of the coal, the efficiency of the coal combustion process and the selection process. It is similar to soil in some of physical and chemical properties, as it contains major, secondary and trace elements, which are found in earth crust. Total major nutrients $\mathrm{N}$ and $\mathrm{P}$ were low i.e. 0.056 and $0.087 \%$, respectively, but it contains sufficient by higher amount of total $\mathrm{K}(0.172 \%), \mathrm{CaO}$ (1.60\%), $\mathrm{MgO}(0.96 \%)$ and total trace elements i.e. Mn 3.98 ppm, Cu 3.60 ppm, Zn $1.30 \mathrm{ppm}$ and $\mathrm{Fe} 3.81 \mathrm{ppm}$, respectively (Bhoyer, 1998).

Presence of organic matter in soil has an additive effect as it reduces the concentration of toxic metals through sorption, lowers the $\mathrm{C} / \mathrm{N}$ ratio and provides organic compounds, which promote microbial proliferation and diversity (Wong and Wong, 1986; Pitchel and Hayes, 1990).

In combination with various organic manure, fly ash can enhance soil microbial activities, nutrient availability and plant productivity (Sikka and Kansal, 1995). Keeping in view the above facts, it an investigation entitled "Consequence of FYM and Fly ash application on the yield, nutrient uptake and quality of Safed musli grown on Vertisol". was carried out during the year 2013-14. 


\section{Materials and Methods}

A field experiment was conducted during 2013-14 at Nagarjun Medicinal Plants Garden, Dr. Panjabrao Deshmukh Krishi Vidyapeeth, Akola (M.S.). There were three levels of FYM and four levels of fly ash with three replications tried in Factorial randomised block design located at between $22^{\circ} 41^{\prime} \mathrm{N}$ latitude and $77^{\circ} 02^{\prime}$ longitudes at an altitude of $307.4 \mathrm{~m}$ above mean sea level and has a subtropical climate.The soil of the experimental site was clayey in nature with pH 8.2 (1:2 soil: water) (Piper, 1996), low in Ec (Piper, 1996), low in organic matter content of $0.5 \%$ (Jackson, 1967), low in available N (Kjeldah's method) (Subbiah and Asija, 1956), low in available P (Jackson, 1967) and medium in available $K$ (Jackson, 1967) at the start of experiment.

The experiment was laid out in a Factorial Randomized Block Design (FRBD) on the same site with three replications having twelve treatment combinations, that is, T1 (control), T2 FYM $\left(0 \mathrm{t} \mathrm{ha}^{-1}\right)+$ Fly ash $(5 \mathrm{t}$ $\left.\mathrm{ha}^{-1}\right)$, T3 (FYM $\left(0 \mathrm{t} \mathrm{ha}^{-1}\right)+$ Fly ash $\left(10 \mathrm{t} \mathrm{ha}^{-1}\right)$, T4 FYM $\left(0 \mathrm{t} \mathrm{ha}^{-1}\right)+$ Fly ash $\left(15 \mathrm{t} \mathrm{ha}^{-1}\right)$, T5 FYM $\left(10 \mathrm{tha}^{-1}\right)+$ Fly ash $\left(0 \mathrm{tha}^{-1}\right)$, T6 FYM $\left(10 \mathrm{t} \mathrm{ha}^{-1}\right)+$ Fly ash $\left(5 \mathrm{t} \mathrm{ha}^{-1}\right)$ T7 FYM $(10 \mathrm{t}$ $\left.\mathrm{ha}^{-1}\right)+$ Fly ash $\left(10 \mathrm{tha}^{-1}\right)$, T8 $\left(\right.$ FYM $\left(10 \mathrm{t} \mathrm{ha}^{-1}\right)$ + Fly ash $\left(15 \mathrm{t} \mathrm{ha}^{-1}\right)$ T9FYM $\left(20 \mathrm{t} \mathrm{ha}^{-1}\right)+$ Fly ash $\left(0 \mathrm{tha}^{-1}\right)$ T10FYM $\left(20 \mathrm{t} \mathrm{ha}^{-1}\right)+$ Fly ash $(5$ $\left.\mathrm{t} \mathrm{ha}^{-1}\right)$ T11 FYM $\left(20 \mathrm{t} \mathrm{ha}^{-1}\right)+$ Fly ash $(10 \mathrm{t}$ $\left.\mathrm{ha}^{-1}\right)$ and T12FYM $\left(20 \mathrm{tha}^{-1}\right)+$ Fly ash (15 t $\mathrm{ha}^{-1}$ ). Planting sprouted roots of Safed musli were used for planting. Two roots bunch was planted at $30 \times 10 \mathrm{~cm}$ spacing on raised beds. The growth observations viz., number of leaves and leaf area were recorded upto 90 days. As after 90 days defoliation of the plants usually occurred in Safed musli. The observations on fresh and dry weight of roots, number of leaves, leaf area and dry matter accumulation at 30, 60, 90 days were recorded.

\section{Statistical analysis}

Standard method of analysis known as 'Analysis of Variance' was applied for the standard analysis of the data, critical difference (CD) at 5 percent level of significance was worked out and use for comparison of different treatment (Gomez and Gomez, 1984).

\section{Results and Discussion}

\section{Effect of FYM and fly ash on number of leaves, leaf area}

Under growth studies, observations on different growth parameters such as number of leaves, leaf area and dry matter accumulation, indicated that the overall growth was found to be increased due to application of FYM and fly ash at various levels. Regarding the effect of FYM on leaf area, the data presented in (Table 1). showed that the effect of FYM on leaf area was found to be significant at 30,60 and 90 DAP. Significantly highest leaf area was recorded with the application of $20 \mathrm{t} \mathrm{FYM} \mathrm{ha}{ }^{-1}\left(\mathrm{M}_{2}\right)$ followed by application of $10 \mathrm{t}$ FYM ha ${ }^{-1}$ $\left(\mathrm{M}_{1}\right)$.This might be due to availability of plant nutrients in addition to improvement in porosity, infiltration through organic manures, resulted into the increased leaf area significantly. The similar results were also reported by Bordia et al., (1995), and Sharma (1996). On the contrary, the fly ash levels had non-significant effect on leaf area recorded during all the growth stages under study except at 60 DAP and significantly highest leaf area $\left(29.56 \mathrm{~cm}^{2}\right)$ was recorded with the application of $15 \mathrm{t}$ fly ash ha ${ }^{-1}$ which was at par with $10 \mathrm{t}$ fly ash ha ${ }^{-1}(\mathrm{~F} 2)$ and $5 \mathrm{t}$ fly ash $\mathrm{ha}^{-1}$ (F1).The incorporation of fly ash in soil was found to be helpful for crop/plant growth was also reported by several workers Warambhe et al., (1991), Jambagi (1995) and Bhople (2006) 


\section{Effect of FYM and fly ash on dry matter accumulation of plant and roots}

The data presented in (Table 2). The dry matter accumulation by plants was non significantly influenced with the application of FYM except at 60 days after planting. Significantly highest dry matter accumulation was recorded with the application of $20 \mathrm{t}$ FYM ha ${ }^{-1}$, however, it was at par with $10 \mathrm{t}$ FYM ha ${ }^{-1}$. Whereas, as dry matter accumulation by roots was found significantly influenced with the application of different levels of FYM at 60 and 90 days after planting. The dry matter accumulation was significantly highest with the application of $20 \mathrm{t} \mathrm{FYM} \mathrm{ha}^{-1}$ however, it was at par with $10 \mathrm{t}$ FYM ha $^{-1}$ at 60 DAP. The significant effect of FYM application on dry matter accumulation might be due to the supply of balanced nutrients to the crop plants. The dry matter accumulation rate was found rapid to very rapid from 30 days growth period upto 60 days, which was on account of increased number of leaves and leaf area. During the growth period of 85 to 90 days, the defoliation started in the crop plant may be the reason for non significant result of FYM application on dry matter accumulation similar results were closely paint by Paturde et al., (2002) while fly ash had significant effect on dry matter accumulation at 60 DAP by plants and at 60 and 90 DAP by roots.

The highest biomass accumulation was observed with $15 \mathrm{t}$ fly ash $\mathrm{ha}^{-1}$ (F3) which was at par with $10 \mathrm{t}$ fly ash $\mathrm{ha}^{-1}$ (F2) and $5 \mathrm{t}$ fly ash $\mathrm{ha}^{-1}$ (F1). Whereas, the dry matter accumulation by roots and plants was non significant at 30 DAP and dry matter accumulation by plants at 90 DAP During the growth period of 85 to 90 days, the defoliation started in the crop plant may be the reason for non significant results of fly ash application on dry matter accumulation.

\section{Effect of FYM and fly ash on root yields}

Application of various levels of FYM and fly ash recorded increased fresh and dry root weight ( $\mathrm{g}$ plant $\left.{ }^{-1}\right)$, fresh and dry root yield of Safed musli. The data presented in (Table 3). The significantly highest root yield was noticed with the application of $20 \mathrm{t} \mathrm{FYM} \mathrm{ha}{ }^{-1}$ and $15 \mathrm{t}$ fly ash $\mathrm{ha}^{-1}$ alone. However, the difference in root yield due to application of 5,10 and $15 \mathrm{t} \mathrm{ha}^{-1}$ were found non significant. The interaction effect of FYM and fly ash on root yield of Safed musli was found non significant. Because of that the use of 10-15 tonnes of FYM ha ${ }^{-1}$ resulted in healthy plant growth ultimately produced highest root yield might be due to the good nutrient status of the substratum similar were closely confirmative by (Bordia et al., 1995).

\section{Effect of FYM and fly ash on quality and protein content of safed musli roots}

The saponin content and yield were significantly increased with the FYM application and highest values were recorded with $20 \mathrm{t} \mathrm{FYM} \mathrm{ha}^{-1}$. The significantly highest saponin content and yield was also recorded with $15 \mathrm{t}$ fly ash $\mathrm{ha}^{-1}$. The significantly highest content of protein was recorded with the application of $20 \mathrm{t} \mathrm{FYM} \mathrm{ha}{ }^{-1}$ followed by $10 \mathrm{t} \mathrm{FYM} \mathrm{ha}^{-1}$. The protein content was also increased with the application of fly ash at various levels, however, the differences among the levels were non significant. The data presented in (Table 4). Besides major nutrients the FYM also contains micronutrients in considerable amount which might have supplied balanced nutrition to the crop plant, which might have resulted in better synthesis of secondary metabolites and increased the saponin content and yield. Similar results were also reported by Bordia et al., (1990), Seth et al., (1991) and Wankhade et al., (2004). 
Table.1 Number of Leaves plant ${ }^{-1}$ and Leaf area plant ${ }^{-1}\left(\mathrm{~cm}^{2}\right)$ as influenced by FYM and fly ash application

\begin{tabular}{|c|c|c|c|c|c|c|}
\hline \multirow[t]{3}{*}{ Treatments } & \multirow{2}{*}{\multicolumn{3}{|c|}{$\begin{array}{c}\text { No. of leaves } \\
\text { Days After planting }\end{array}$}} & \multirow{2}{*}{\multicolumn{3}{|c|}{$\begin{array}{c}\text { Leaf area } \\
\text { Days After planting }\end{array}$}} \\
\hline & & & & & & \\
\hline & 30 & 60 & 90 & 30 & 60 & 90 \\
\hline \multicolumn{7}{|c|}{ FYM levels $\left(\mathrm{t} \mathrm{ha}^{-1}\right)$} \\
\hline M0 (0) & 9.92 & 16.00 & 14.08 & 22.33 & 27.58 & 25.58 \\
\hline M1 (10) & 10.25 & 15.75 & 15.42 & 23.58 & 29.42 & 27.33 \\
\hline M2 (20) & 10.92 & 15.75 & 15.92 & 23.25 & 30.17 & 27.33 \\
\hline $\mathrm{SE}(\mathrm{m}) \pm$ & 0.38 & 0.26 & 0.23 & 0.30 & 0.21 & 0.28 \\
\hline CD at $5 \%$ & NS & NS & NS & 0.88 & 0.60 & 0.83 \\
\hline \multicolumn{7}{|c|}{ Fly ash levels ( $\left.\mathrm{tha}^{-1}\right)$} \\
\hline FO (0) & 9.89 & 15.44 & 14.11 & 23.00 & 28.33 & 26.56 \\
\hline F1 (5) & 10.00 & 16.33 & 15.22 & 23.22 & 29.00 & 26.44 \\
\hline F2 (10) & 10.89 & 16.11 & 15.44 & 23.00 & 29.33 & 27.00 \\
\hline F3 (15) & 10.67 & 15.44 & 15.78 & 23.00 & 29.56 & 27.00 \\
\hline $\mathrm{SE}(\mathrm{m}) \pm$ & 0.44 & 0.30 & 0.27 & 0.35 & 0.24 & 0.33 \\
\hline CD at $5 \%$ & NS & NS & $\mathrm{NS}$ & NS & 0.69 & $\mathrm{NS}$ \\
\hline \multicolumn{7}{|c|}{ Interaction effect (FYM x Fly ash) } \\
\hline $\mathbf{S E}(\mathbf{m}) \pm$ & 0.75 & 0.52 & 0.46 & 0.60 & 0.41 & 0.57 \\
\hline CD at $5 \%$ & NS & NS & NS & NS & NS & NS \\
\hline
\end{tabular}

Table.2 Dry Matter accumulation ( $\mathrm{q} \mathrm{ha}^{-1}$ ) as influenced by FYM and fly ash application

\begin{tabular}{|c|c|c|c|c|c|c|}
\hline \multirow[t]{3}{*}{ Treatments } & \multirow{2}{*}{\multicolumn{3}{|c|}{$\begin{array}{l}\text { Plant Leaf biomass } \\
\text { Days after planting }\end{array}$}} & \multirow{2}{*}{\multicolumn{3}{|c|}{$\begin{array}{c}\text { Root biomass } \\
\text { Days after planting }\end{array}$}} \\
\hline & & & & & & \\
\hline & 30 & 60 & 90 & 30 & 60 & 90 \\
\hline \multicolumn{7}{|c|}{ FYM levels $\left(\mathrm{t} \mathrm{ha}^{-1}\right)$} \\
\hline M0 (0) & 1.28 & 1.58 & 1.47 & 2.84 & 2.88 & 3.61 \\
\hline M1 (10) & 1.32 & 1.63 & 1.53 & 3.02 & 3.78 & 4.85 \\
\hline M2 (20) & 1.34 & 1.64 & 1.60 & 3.16 & 3.88 & 6.34 \\
\hline $\mathrm{SE}(\mathbf{m}) \pm$ & 0.01 & 0.01 & 0.04 & 0.10 & 0.11 & 0.08 \\
\hline CD at $5 \%$ & NS & 0.03 & NS & NS & 0.33 & 0.24 \\
\hline \multicolumn{7}{|c|}{ Fly ash levels $\left(\mathrm{t} \mathrm{ha}^{-1}\right)$} \\
\hline F0 (0) & 1.30 & 1.59 & 1.53 & 2.94 & 3.24 & 4.42 \\
\hline F1 (5) & 1.32 & 1.62 & 1.55 & 3.00 & 3.26 & 4.99 \\
\hline F2 (10) & 1.33 & 1.62 & 1.54 & 3.05 & 3.76 & 5.1 \\
\hline F3 (15) & 1.31 & 1.65 & 1.52 & 3.04 & 3.79 & 5.23 \\
\hline $\mathrm{SE}(\mathrm{m}) \pm$ & 0.01 & 0.01 & 0.04 & 0.12 & 0.13 & 0.09 \\
\hline CD at $5 \%$ & NS & 0.03 & NS & $\mathrm{NS}$ & 0.38 & 0.27 \\
\hline \multicolumn{7}{|c|}{ Interaction effect (FYM x Fly ash) } \\
\hline $\mathrm{SE}(\mathrm{m}) \pm$ & 0.03 & 0.02 & 0.08 & 0.2 & 0.22 & 0.16 \\
\hline CD at $5 \%$ & NS & NS & NS & NS & NS & NS \\
\hline
\end{tabular}


Table.3 Root yield of safed musli as influenced by FYM and fly ash application

\begin{tabular}{|c|c|c|c|c|}
\hline Treatments & $\begin{array}{c}\text { Fresh wt of } \\
\text { root } \\
\left(\mathrm{g} \text { plant }^{-1}\right)\end{array}$ & $\begin{array}{l}\text { Dry wt of root } \\
\quad\left(\text { g plant }^{-1}\right)\end{array}$ & $\begin{array}{l}\text { Fresh wt of } \\
\operatorname{root}\left(q \mathrm{ha}^{-1}\right)\end{array}$ & $\begin{array}{c}\text { Dry wt of } \\
\text { root } \\
\left(q \text { ha }^{-1}\right)\end{array}$ \\
\hline \multicolumn{5}{|l|}{ FYM levels $\left(\mathrm{t} \mathrm{ha}^{-1}\right)$} \\
\hline M0 (0) & 9.95 & 1.81 & 24.00 & 4.36 \\
\hline M1 (10) & 12.79 & 2.32 & 30.83 & 5.60 \\
\hline M2 (20) & 16.18 & 2.94 & 39.00 & 7.09 \\
\hline $\mathrm{SE}(\mathrm{m}) \pm$ & 0.18 & 0.03 & 0.44 & 0.08 \\
\hline CD at $5 \%$ & 0.54 & 0.10 & 1.30 & 0.24 \\
\hline \multicolumn{5}{|l|}{ Fly ash levels $\left(\mathrm{t} \mathrm{ha}^{-1}\right)$} \\
\hline F0 (0) & 11.80 & 2.15 & 28.46 & 5.17 \\
\hline F1 (5) & 13.08 & 2.38 & 31.55 & 5.74 \\
\hline F2 (10) & 13.35 & 2.43 & 32.19 & 5.85 \\
\hline F3 (15) & 13.65 & 2.48 & 32.91 & 5.98 \\
\hline $\mathrm{SE}(\mathrm{m}) \pm$ & 0.21 & 0.04 & 0.51 & 0.09 \\
\hline CD at $5 \%$ & 0.62 & 0.11 & 1.50 & 0.27 \\
\hline \multicolumn{5}{|c|}{ Interaction effect (FYM x Fly ash) } \\
\hline $\mathrm{SE}(\mathrm{m}) \pm$ & 0.37 & 0.07 & 0.89 & 0.16 \\
\hline CD at $5 \%$ & NS & NS & NS & NS \\
\hline
\end{tabular}

Table.4 Quality of safed musli roots as influenced by FYM and fly ash application

\begin{tabular}{|c|c|c|c|}
\hline Treatments & Saponin content (\%) & Saponin yield $\left(\mathrm{kg} \mathrm{ha}^{-1}\right)$ & $\begin{array}{c}\text { Protein content } \\
(\%)\end{array}$ \\
\hline \multicolumn{4}{|l|}{ FYM levels $\left(\mathrm{tha}^{-1}\right)$} \\
\hline M0 (0) & 7.19 & 31.45 & 7.93 \\
\hline M1 (10) & 7.97 & 44.72 & 8.22 \\
\hline M2 (20) & 8.11 & 57.48 & 8.50 \\
\hline $\mathrm{SE}(\mathbf{m}) \pm$ & 0.01 & 0.64 & 0.06 \\
\hline CD at $5 \%$ & 0.03 & 1.88 & 0.18 \\
\hline \multicolumn{4}{|l|}{ Fly ash levels $\left(\mathrm{t} \mathrm{ha}^{-1}\right)$} \\
\hline Fo (0) & 7.57 & 39.96 & 8.04 \\
\hline F1 (5) & 7.70 & 44.48 & 8.26 \\
\hline F2 (10) & 7.76 & 45.61 & 8.23 \\
\hline F3 (15) & 8.00 & 48.16 & 8.33 \\
\hline $\mathrm{SE}(\mathbf{m}) \pm$ & 0.01 & 0.74 & 0.06 \\
\hline CD at $5 \%$ & 0.03 & 2.17 & 0.19 \\
\hline \multicolumn{4}{|c|}{ Interaction effect (FYM x Fly ash) } \\
\hline $\mathrm{SE}(\mathrm{m}) \pm$ & 0.03 & 1.28 & 0.11 \\
\hline CD at $5 \%$ & 0.08 & 3.75 & NS \\
\hline
\end{tabular}


Table.5 Nitrogen (\%) content and uptake in plant and root as influenced by FYM and fly ash application

\begin{tabular}{|c|c|c|c|c|c|c|c|c|c|c|c|c|c|c|c|}
\hline \multirow{3}{*}{$\begin{array}{l}\text { Treatments } \\
\text { FYM levels }\left(\mathrm{t} \mathrm{ha}^{-1}\right)\end{array}$} & \multirow{2}{*}{\multicolumn{3}{|c|}{$\begin{array}{l}\text { Nitrogen content in } \\
\text { plants } \\
\text { Days after planting }\end{array}$}} & \multirow{2}{*}{\multicolumn{3}{|c|}{$\begin{array}{c}\text { Nitrogen content in } \\
\text { roots } \\
\text { Days after } \\
\text { planting }\end{array}$}} & \multirow{2}{*}{\multicolumn{3}{|c|}{$\begin{array}{c}\text { Nitrogen uptake by } \\
\text { plants }\end{array}$}} & \multirow{2}{*}{\multicolumn{3}{|c|}{$\begin{array}{c}\text { Nitrogen uptake } \\
\text { by roots } \\
\text { Days after } \\
\text { planting }\end{array}$}} & \multicolumn{3}{|c|}{ Total uptake } \\
\hline & & & & & & & & & & & & & \multicolumn{3}{|c|}{$\begin{array}{c}\text { Days after } \\
\text { Planting }\end{array}$} \\
\hline & 30 & 60 & 90 & 30 & 60 & 90 & 30 & 60 & 90 & 30 & 60 & 90 & 30 & 60 & 90 \\
\hline M0 (0) & 2.09 & 1.79 & 1.74 & 2.82 & 2.12 & 1.66 & 5.92 & 5.06 & 2.57 & 8.00 & 6.11 & 5.96 & 13.92 & 11.17 & 8.53 \\
\hline M1 (10) & 2.36 & 1.86 & 1.81 & 3.03 & 2.33 & 1.74 & 7.13 & 5.63 & 2.76 & 9.17 & 8.92 & 8.42 & 16.3 & 14.55 & 11.18 \\
\hline M2 (20) & 2.67 & 1.94 & 1.86 & 3.20 & 2.50 & 2.00 & 8.41 & 6.20 & 2.96 & 10.10 & 9.71 & 12.68 & 18.51 & 15.91 & 15.64 \\
\hline $\mathrm{SE}(\mathrm{m}) \pm$ & 0.05 & 0.01 & 0.01 & 0.02 & 0.06 & 0.07 & 0.31 & 0.08 & 0.07 & 0.32 & 0.38 & 0.35 & 0.63 & 0.46 & 0.42 \\
\hline CD at $5 \%$ & 0.14 & 0.03 & 0.03 & 0.06 & 0.17 & 0.21 & 0.90 & 0.24 & 0.20 & 0.92 & 1.11 & 1.03 & 1.82 & 1.35 & 1.23 \\
\hline F0 (0) & 2.34 & 1.84 & 1.79 & 2.98 & 2.28 & 1.73 & 6.92 & 5.40 & 2.74 & 8.77 & 7.50 & 7.74 & 15.69 & 12.9 & 10.48 \\
\hline F1 (5) & 2.37 & 1.86 & 1.80 & 3.01 & 2.31 & 1.79 & 7.13 & 5.63 & 2.79 & 9.06 & 7.71 & 9.07 & 16.19 & 13.34 & 11.86 \\
\hline F2 (10) & 2.38 & 1.86 & 1.80 & 3.04 & 2.34 & 1.83 & 7.27 & 5.65 & 2.78 & 9.27 & 8.82 & 9.49 & 16.54 & 14.47 & 12.27 \\
\hline F3 (15) & 2.40 & 1.88 & 1.81 & 3.05 & 2.35 & 1.83 & 7.31 & 5.83 & 2.75 & 9.28 & 8.96 & 9.77 & 16.59 & 14.79 & 12.52 \\
\hline $\mathrm{SE}(\mathrm{m}) \pm$ & 0.06 & 0.01 & 0.01 & 0.02 & 0.07 & 0.08 & 0.35 & 0.10 & 0.08 & 0.36 & 0.44 & 0.41 & 0.71 & 0.54 & 0.49 \\
\hline CD at $5 \%$ & NS & NS & NS & NS & NS & NS & NS & 0.28 & NS & NS & NS & 1.19 & NS & NS & NS \\
\hline \multicolumn{16}{|c|}{$\begin{array}{l}\text { Interaction effect (FYM x } \\
\text { Fly ash) }\end{array}$} \\
\hline $\mathrm{SE}(\mathrm{m}) \pm$ & 0.10 & 0.02 & 0.01 & 0.04 & 0.12 & 0.15 & 0.61 & 0.16 & 0.13 & 0.63 & 0.76 & 0.70 & 1.24 & 0.92 & 0.83 \\
\hline CD at $5 \%$ & NS & NS & NS & NS & NS & NS & NS & NS & NS & NS & NS & NS & $\mathrm{NS}$ & $\mathrm{NS}$ & NS \\
\hline
\end{tabular}


Table.6 Phosphorous content and uptake $\left(\mathrm{kg} \mathrm{ha}^{-1}\right)$ by safed musli as influenced by FYM and Fly ash application

\begin{tabular}{|c|c|c|c|c|c|c|c|c|c|c|c|c|c|c|c|}
\hline \multirow{3}{*}{$\begin{array}{l}\text { Treatments } \\
\text { FYM levels }\left(\mathbf{t h a}^{-1}\right)\end{array}$} & \multirow{2}{*}{\multicolumn{3}{|c|}{$\begin{array}{l}\text { Phosphorous } \\
\text { content in plants } \\
\text { Days after } \\
\text { planting }\end{array}$}} & \multirow{2}{*}{\multicolumn{3}{|c|}{$\begin{array}{l}\text { Phosphorous } \\
\text { content in } \\
\text { roots } \\
\text { Days after } \\
\text { planting }\end{array}$}} & \multirow{2}{*}{\multicolumn{3}{|c|}{$\begin{array}{l}\text { Phosphorous } \\
\text { uptake by plant } \\
\text { Days after }\end{array}$}} & \multirow{2}{*}{\multicolumn{3}{|c|}{$\begin{array}{c}\text { Phosphorous uptake } \\
\text { by root } \\
\text { Days after planting }\end{array}$}} & \multicolumn{3}{|c|}{$\begin{array}{c}\text { Total uptake } \\
\text { of } P\end{array}$} \\
\hline & & & & & & & & & & & & & Da & after & nting \\
\hline & 30 & 60 & 90 & 30 & 60 & 90 & 30 & 60 & 90 & 30 & 60 & 90 & 30 & 60 & 90 \\
\hline M0 (0) & 0.14 & 0.26 & 0.18 & 0.11 & 0.17 & 0.21 & 0.18 & 0.42 & 0.27 & 0.31 & 0.48 & 0.76 & 0.49 & 0.9 & 1.03 \\
\hline M1 (10) & 0.18 & 0.36 & 0.23 & 0.15 & 0.23 & 0.25 & 0.24 & 0.58 & 0.36 & 0.46 & 0.86 & 1.23 & 0.7 & 1.44 & 1.59 \\
\hline M2 (20) & 0.23 & 0.51 & 0.32 & 0.22 & 0.26 & 0.32 & 0.30 & 0.83 & 0.51 & 0.70 & 1.01 & 2.04 & 1.00 & 1.84 & 2.55 \\
\hline $\mathrm{SE}(\mathrm{m}) \pm$ & 0.01 & 0.01 & 0.02 & 0.02 & 0.05 & 0.02 & 0.01 & 0.02 & 0.03 & 0.07 & 0.03 & 0.13 & 0.08 & 0.05 & 0.16 \\
\hline CD at $5 \%$ & 0.03 & 0.03 & 0.06 & 0.06 & NS & 0.07 & 0.03 & 0.06 & 0.09 & 0.19 & 0.09 & 0.38 & 0.22 & 0.15 & 0.47 \\
\hline \multicolumn{16}{|l|}{ Fly ash levels $\left(\mathrm{t} \mathrm{ha}^{-1}\right)$} \\
\hline F0 (0) & 0.17 & 0.35 & 0.24 & 0.15 & 0.21 & 0.26 & 0.23 & 0.55 & 0.37 & 0.46 & 0.71 & 1.22 & 0.69 & 1.26 & 1.59 \\
\hline F1 (5) & 0.19 & 0.37 & 0.24 & 0.16 & 0.22 & 0.26 & 0.25 & 0.60 & 0.38 & 0.48 & 0.72 & 1.33 & 0.73 & 1.32 & 1.71 \\
\hline F2 (10) & 0.18 & 0.38 & 0.25 & 0.16 & 0.22 & 0.26 & 0.24 & 0.62 & 0.38 & 0.50 & 0.84 & 1.38 & 0.74 & 1.46 & 1.76 \\
\hline F3 (15) & 0.19 & 0.40 & 0.25 & 0.17 & 0.22 & 0.26 & 0.25 & 0.67 & 0.38 & 0.53 & 0.86 & 1.43 & 0.78 & 1.53 & 1.81 \\
\hline $\mathrm{SE}(\mathbf{m}) \pm$ & 0.01 & 0.02 & 0.02 & 0.02 & 0.06 & 0.03 & 0.01 & 0.02 & 0.04 & 0.08 & 0.04 & 0.15 & 0.09 & 0.06 & 0.19 \\
\hline CD at $5 \%$ & NS & NS & NS & NS & NS & NS & NS & 0.07 & NS & NS & 0.11 & NS & NS & 0.18 & NS \\
\hline \multicolumn{16}{|c|}{ Interaction effect (FYM x Fly ash) } \\
\hline $\mathrm{SE}(\mathbf{m}) \pm$ & 0.01 & 0.03 & 0.04 & 0.04 & 0.11 & 0.05 & 0.02 & 0.04 & 0.06 & 0.13 & 0.06 & 0.26 & 0.15 & 0.1 & 0.32 \\
\hline CD at $5 \%$ & NS & $\mathrm{NS}$ & $\mathrm{NS}$ & NS & NS & NS & NS & $\mathrm{NS}$ & NS & $\mathrm{NS}$ & NS & NS & NS & NS & NS \\
\hline
\end{tabular}


Table.7 Potassium content and uptake $\left(\mathrm{kg} \mathrm{ha}^{-1}\right)$ by safed musli as influenced by FYM and Fly ash application

\begin{tabular}{|c|c|c|c|c|c|c|c|c|c|c|c|c|c|c|c|}
\hline \multirow[t]{3}{*}{ Treatments } & \multicolumn{3}{|c|}{$\begin{array}{c}\text { Potassium content in } \\
\text { plants }\end{array}$} & \multicolumn{3}{|c|}{$\begin{array}{c}\text { Potassium content in } \\
\text { roots }\end{array}$} & \multicolumn{3}{|c|}{$\begin{array}{c}\text { Potassium uptake } \\
\text { by plants }\end{array}$} & \multicolumn{3}{|c|}{$\begin{array}{c}\text { Potassium uptake } \\
\text { by } \\
\text { roots }\end{array}$} & \multicolumn{3}{|c|}{ Total uptake of K } \\
\hline & \multicolumn{3}{|c|}{ Days after planting } & \multicolumn{3}{|c|}{ Days after planting } & \multicolumn{3}{|c|}{ Days after planting } & \multicolumn{3}{|c|}{$\begin{array}{l}\text { Days after } \\
\text { planting }\end{array}$} & \multicolumn{3}{|c|}{$\begin{array}{l}\text { Days after } \\
\text { planting }\end{array}$} \\
\hline & 30 & 60 & 90 & 30 & 60 & 90 & 30 & 60 & 90 & 30 & 60 & 90 & 30 & 60 & 90 \\
\hline \multicolumn{16}{|c|}{ FYM levels $\left(\mathrm{t} \mathrm{ha}^{-1}\right)$} \\
\hline M0 (0) & 1.28 & 1.54 & 1.44 & 1.06 & 1.34 & 1.44 & 1.63 & 2.44 & 2.13 & 3.02 & 3.84 & 5.20 & 4.65 & 6.28 & 7.33 \\
\hline M1 (10) & 1.36 & 1.72 & 1.52 & 1.15 & 1.42 & 1.70 & 1.80 & 2.81 & 2.33 & 3.47 & 5.37 & 8.24 & 5.27 & 8.18 & 10.57 \\
\hline M2 (20) & 1.48 & 1.81 & 1.56 & 1.19 & 1.69 & 1.80 & 1.99 & 2.98 & 2.49 & 3.75 & 6.55 & 11.40 & 5.74 & 9.53 & 13.89 \\
\hline SE(m) \pm & 0.02 & 0.01 & 0.01 & 0.01 & 0.02 & 0.01 & 0.04 & 0.02 & 0.06 & 0.13 & 0.19 & 0.13 & 0.17 & 0.21 & 0.19 \\
\hline CD at $5 \%$ & 0.06 & 0.03 & 0.03 & 0.03 & 0.07 & 0.03 & 0.11 & 0.06 & 0.17 & 0.37 & 0.56 & 0.39 & 0.48 & 0.62 & 0.56 \\
\hline \multicolumn{16}{|c|}{ Fly ash levels $\left(t^{h a^{-1}}\right)$} \\
\hline F0 (0) & 1.34 & 1.68 & 1.50 & 1.12 & 1.46 & 1.62 & 1.75 & 2.67 & 2.30 & 3.30 & 4.85 & 7.36 & 5.05 & 7.52 & 9.66 \\
\hline F1 (5) & 1.37 & 1.69 & 1.51 & 1.13 & 1.48 & 1.64 & 1.82 & 2.75 & 2.34 & 3.40 & 4.87 & 8.32 & 5.22 & 7.62 & 10.66 \\
\hline F2 (10) & 1.38 & 1.70 & 1.51 & 1.14 & 1.48 & 1.66 & 1.84 & 2.75 & 2.33 & 3.47 & 5.58 & 8.57 & 5.31 & 8.33 & 10.90 \\
\hline F3 (15) & 1.40 & 1.70 & 1.51 & 1.14 & 1.50 & 1.66 & 1.83 & 2.80 & 2.30 & 3.48 & 5.71 & 8.88 & 5.31 & 8.51 & 11.18 \\
\hline $\mathrm{SE}(\mathrm{m}) \pm$ & 0.02 & 0.01 & 0.01 & 0.01 & 0.03 & 0.01 & 0.04 & 0.02 & 0.07 & 0.15 & 0.22 & 0.15 & 0.19 & 0.24 & 0.22 \\
\hline CD at $5 \%$ & NS & NS & NS & NS & NS & NS & NS & 0.07 & NS & NS & 0.65 & 0.45 & NS & 0.72 & NS \\
\hline \multicolumn{16}{|c|}{ Interaction effect (FYM x Fly ash) } \\
\hline $\mathrm{SE}(\mathrm{m}) \pm$ & 1.34 & 1.68 & 1.50 & 1.12 & 1.46 & 1.62 & 0.07 & 0.04 & 0.11 & 0.25 & 0.39 & 0.26 & 0.32 & 0.43 & 0.37 \\
\hline CD at $5 \%$ & 1.37 & 1.69 & 1.51 & 1.13 & 1.48 & 1.64 & NS & NS & NS & NS & NS & NS & NS & NS & NS \\
\hline
\end{tabular}


Effect of FYM and Fly ash content and uptake of nutrient in safed-musali

The content and uptake of nutrients $(\mathrm{N}, \mathrm{P}$, $\mathrm{K}$,), were also significantly increased with the increasing levels of FYM. The data presented in (Table 5, 6, 7). However, the content of macro $(\mathrm{N}, \mathrm{P}$ and $\mathrm{K}$ ) nutrients in crop plants was non significant with application of different level of fly ash.

Whereas, application of FYM @ $20 \mathrm{t} \mathrm{ha}^{-1}$, and fly ash@15 t fly ash ha ${ }^{-1}$ alone significantly increased the content and uptake of micronutrients by safed musli. It might be due to application of FYM to the soil, the nutrients are released slowly and available to the plant over a long period of time, resulted in the increase content and uptake of macro and micronutrient at all the growth stages.

The increased content and uptake of nutrient due to FYM application is also reported by Chaurasia and Singh (1993), Joy et al., (2005) Bhaisare et al., (2000) also reported significantly highest content and uptake with the increasing levels of fly ash upto $10 \mathrm{tha}^{-1}$

It may be concluded that from the present investigation, it is revealed that the application of $20 \mathrm{t}$ FYM ha ${ }^{-1}$ recorded significantly highest growth, content and uptake of nutrients, root yield, saponin and protein content and saponin yield.

The content and uptake of nutrients, root yield and saponin content and its yield were recorded significantly highest with the application of fly ash @ $15 \mathrm{t} \mathrm{ha}^{-1}$. However, it was at par with $10 \mathrm{t}$ and $5 \mathrm{t}$ fly ash ha ${ }^{1}$.Therefore, it is concluded that the application of FYM @ $20 \mathrm{t}$ and fly ash @ $5 \mathrm{t}$ $\mathrm{ha}^{-1}$ was found beneficial way of nutrient management for Safed musli to enhance the root yield and quality.

\section{References}

Bhoyar, S. M., 1998. Effect of fly ash application on micronutrient status of black soil in multiple cropping system. Ph.D. Thesis (unpub.) Dr. PDKV Akola

Bhaisare Bharti, D.B., Matte, W.P. Badole and Anjali Deshmukh., 2000. Effect of fly ash on yield, uptake of nutrient and quality of green gram grown on vertisol. J. Soils and crop. 10(1):122124

Bhople, B.S. 2006 Growth, nutrient uptake and yield of sunflower as influenced by the application of graded levels of fly ash to black soil. M.Sc. Thesis (unpub), Dr. PDKV Akola (M.S)

Bordia, P.C., 1992. Natural farming of wonder herb safed musli (Chlorophytum spp.). Proc. Nat. Seminar Natural Farming. Pp. 69-77.

Bordia, P.C., A. Joshi and M.M. Simlot 1995. Safed musli. Advances in Horticulture Medicinal and Aromatic Plant. 11: 439.

Bordia, P.C., P. Seth and M.M. Simlot 1990. Exploration of safed musli (Chlorophytum sp.) in the Araivali region and preliminary observation. Paper presented in the national symposium on conservation and university of living resources. University of Agriculture Sciences, GKVK, Bangalore. Pp. 4.

Chaurasia, S.N., and K.P. Singh, 1993. Effect of nitrogen levels and haulms cutting date on nutrient concentration and uptake in different plant parts of potato. J. Indian Potato Ass. 20(2): 169-171.

Gomeze, K.A., and A.A. Gomez. 1984. Statistical procedure for Agricultural Research $2^{\text {nd }}$ edition.

Jackson, M.L., 1967. Soil chemical analysis prentice Hall of India, Pvt. Ltd., New 
Delhi.

Joy, P.P., K.E., Savithri, S.Mathew, J. Thomas, and K. Kurein, 2005. Effect of sole and combined application of FYM and fertilizer on growth, yield and quality of black musli (Curligo orchioides). Journal of Medicinal and Aromatic Plant Sciences, 27: 454-461

Kirtikar, K.R., and B.D. Basu. 1975. Indian Medicinal Plants. 4 ( $2^{\text {nd }} E d$. $)$.

Paturde, J.T., Wankhade, S. G., Khode, P. P., Chatol, P. U., Deo, D. D. And Bhuyar, S. A., 2002, Effect of organic manures and plant population on yield of Safed musli (Chlorophytum borivilianum). Agricultural Sciences Digest, 22(1): 51-52.

Piper, C.S., 1996. Soil and plant analysis $4^{\text {th }}$ Edn. Inc. Pub., New York: 135-200.

Seth, P., M.K Sharma and M.M Simlot (1991): Saponi in chlorophytum spp. Paper presented in diamond jubilee annual journal body meeting of society of Biological chemist of India and Biotech, Institute of chemical biological, Calcutta. 27-30 Dec. Abstr. No. 028.

Sharma, 1996. Effect of FYM on yield viability of safed musli grown under varying plant population, AICRP on Medicinal and Aromatic Plant. 11 ${ }^{\text {th }}$ All India Workshop held at Rajasthan Agril. University, Udaipur: 129

Wankhade, S.G. P.P. Khode and J.T. Paturde, 2004. Effect of organic manure and fertilizer on the yield and quality of safed musli. PKV Res. J. 28(1):111112.

Warambhe, P.E., 1991. Effect of fly ash application on growth, yield and nutrient uptake of cotton. M.Sc. Thesis (Unpub.), Dr. PDKV, Akola (M.S.).

\section{How to cite this article:}

Payal B. Hadke, S.G. Wankhade and Sagar N. Ingle. 2019. Consequence of Fym and Fly Ash Application on Yield, Nutrient Uptake, and Quality of Safed Musli Grown on Inceptisols. Int.J.Curr.Microbiol.App.Sci. 8(05): 263-273. doi: https://doi.org/10.20546/ijcmas.2019.805.030 